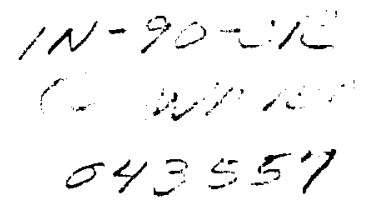

\title{
RADIATIVE INSTABILITIES IN THREE-DIMENSIONAL ASTROPHYSICAL MASERS
}

\author{
Gerardo A. SCapPaticCi \\ Department of Physical Sciences, San Diego City College, San Diego, CA 92101 \\ AND \\ WILLIAM D. WATSON \\ Department of Physics, University of Illinois at Urbana-Champaign, Urbana, IL 61801-3080 \\ Received 1994 August 8; accepted 1995 February 7
}

\begin{abstract}
Inherent instabilities in the radiative transfer for astrophysical masers have been recognized and calculated in the linear maser idealization in our previous investigations. The same instabilities are now shown to occur in the more realistic, three-dimensional geometries. Fluctuations in the emergent flux result and may be related to the observed fluctuations in the radiative flux from the $1665 \mathrm{MHz} \mathrm{OH}$ masers that have been reported to occur on timescales as short as $1000 \mathrm{~s}$. The time-dependent differential equations of radiative transfer are solved numerically for three-dimensional astrophysical masers. Computations are performed for spherical and elongated (rectangular parallelepiped) geometries.
\end{abstract}

Subject headings: instabilities - masers — radiative transfer

\section{INTRODUCTION}

Clegg \& Cordes (1991) have reported fluctuations of $\sim 10 \%$ in the flux of radiation from interstellar $1665 \mathrm{MHz} \mathrm{OH}$ masers on timescales down to $1000 \mathrm{~s}$. Calculations have been performed in which inherent instabilities in the radiative transfer are found to be a likely cause for the observed fluctuations (Scappaticci \& Watson 1992a, b; hereafter SW1 and SW2). The occurrence of the instabilities has, however, been demonstrated only in the usual idealization of a linear maser. In the linear maser idealization, all rays of maser radiation follow the same path through the maser. In actual astrophysical masers, the rays are separated by distances of $10^{13}-10^{14} \mathrm{~cm}$ - the observed dimensions of the masers. For significant fluctuations in the observed maser flux, the entire cross section of the masing region (or at least a large fraction) must respond collectively to the instabilities. It may not be evident from the investigations to date that such collective behavior is a consequence of the instabilities that have been calculated for linear masers. The purpose of this paper is the demonstration of these instabilities for the radiative transfer in three-dimensional masers as represented by spheres and cylinders. Although the range of the values for the relevant parameters in which the masers are unstable (both linear and three-dimensional) has so far been found to be limited to those that are plausible for the 1665 $\mathrm{MHz}$ and other $18 \mathrm{~cm} \mathrm{OH}$ masers, the basic instability may appear in other forms. Rapid time variations of the flux is a pervasive feature of astrophysical masers (e.g., Argon et al. 1994).

In SW1 and SW2, the instabilities were established both by a linear stability analysis and by numerically integrating the full, partial differential equations in space and time. The instabilities were evident in both for exactly the same range of values for the relevant parameters. This indicates that either procedure is reliable. For the spheres and cylinders considered in this paper, only the results of the numerical integration of the full equations are used to examine the instabilities. They are found to occur at essentially the same range of parameter values as for linear masers-a result that tends to be reassuring about the reliability of the calculations presented in $\S 3$.

\section{BASIC METHODS}

The time-dependent equations of radiative transfer in three dimensions are solved by generalizing our previous methods for a linear maser in a straightforward manner. The masing transition is treated in the common, two-level approximation in which the interaction with other molecular states is approximated by "phenomenological" pumping rates $\Lambda$ and decay rates $\Gamma$. With the usual Rayleigh-Jeans approximation for the maser radiation, the time-dependent rate equation for the difference per unit volume between the populations of the upper $(u)$ and lower $(l)$ states of the masing transitions is given by

$$
\frac{\partial \rho}{\partial t}=\frac{\Gamma L}{c}\left[1-\left(1+\beta^{\prime} \int \boldsymbol{T} \cdot d \boldsymbol{\Omega}-\frac{A}{\Gamma}\right) \rho-\frac{A}{\Gamma} \rho_{s}\right],
$$

where

$$
\rho(\tilde{\boldsymbol{x}}, \tilde{t})=\Gamma\left(n_{u}-n_{l}\right) / \Delta \Lambda
$$

is the normalized difference between the populations per magnetic substate of the upper $\left(n_{u}\right)$ and lower $\left(n_{l}\right)$ states at location $x$ and time $t, \Delta \Lambda=\Lambda_{u}-\Lambda_{l}, A$ is the Einstein coefficient for spontaneous emission,

$$
\beta^{\prime}=\frac{1}{2 \pi} \frac{k T_{0}}{h v} \frac{A}{\Gamma}
$$

$v$ is the frequency of the maser transition, and

$$
\rho_{s}=\Gamma\left(n_{u}+n_{l}\right) / \Delta \Lambda=\left(\Lambda_{u}+\Lambda_{l}\right) / \Delta \Lambda=\text { constant } .
$$

The integral of the normalized brightness temperature $\tilde{\boldsymbol{T}}(\tilde{\boldsymbol{x}}, \tilde{t})$ of the maser radiation over all angles at location $\tilde{\boldsymbol{x}}$ in equation (1) gives the effect of stimulated transitions on the populations. Normalized time and spatial coordinates $\tilde{t}=t /(L / c)$ and $\tilde{\boldsymbol{x}}=$ $\boldsymbol{x} / L$ are expressed in terms of the characteristic distance $L$ which is the length for cylinders or the diameter for spheres. Incident continuum radiation has a brightness temperature $T_{0}$. The equation of radiative transfer for the intensity of maser radiation expressed as a normalized brightness temperature 
: 
$\tilde{\boldsymbol{T}}(\tilde{\boldsymbol{x}}, \tilde{t})$ is then

$$
\frac{\partial \tilde{T}}{\partial \tilde{s}}+\frac{\partial \tilde{T}}{\partial \tilde{t}}=\tau\left(\rho \tilde{T}+\frac{h v}{2 k T_{0}} \rho+\frac{h v}{2 k T_{0}} \rho_{s}\right)
$$

where $\tilde{s}$ is the normalized distance along the path of the ray of radiation and

$$
\tau=g_{u}\left(\frac{c^{3}}{8 \pi v^{3}}\right) \frac{A}{\Delta v} \frac{\Delta \Lambda}{\Gamma} L,
$$

and $\tilde{\boldsymbol{T}}(\tilde{\boldsymbol{x}}, \tilde{t})$ is the actual brightness temperature divided by $T_{0}$.

As in the previous calculations, a rectangular distribution of width $\Delta v$ in velocity is adopted for the molecular populations and an analogous rectangular profile is adopted for the maser radiation. Rapid relaxation of the molecular velocities is thus assumed. Computations are then performed at only a single velocity in equation (1) and frequency in equation (5). These approximations were also made in the previous discussions and were discussed at that time. Subsequently, computations have been performed for a linear maser in which the Maxwellian distribution for the particle velocities is retained. The distribution in frequency for $\tilde{T}$ is then obtained as a result of solving equation (5) at a sufficient number of frequencies to define the spectral line profile. The effect of these changes on the stability of the masers is negligible (Emmering \& Watson 1994).

To perform the computations, we proceed in the following manner. Locations are specified at which the population differences $\rho(\tilde{\boldsymbol{x}}, t)$ are to be computed from equation (1). A separate group of rays is then specified for each such location in order to compute the integral of the intensity over angles that is required in equation (1). A group of such rays is thus "centered upon " the location at which the group is used for computing $\rho(\tilde{\boldsymbol{x}}, t)$. That is, each member of the group passes through that location and the directions of the rays are chosen for optimal efficiency in computing the integral. These directions are, in general, different for each location. Equation (5) is used to compute the intensities along these rays as a function of time and as a function of the distance along the rays by taking steps $\Delta s=c \Delta t$-exactly as was done in SW2. Here there are a large number of rays, whereas in SW2 there were only two rays - the "forward" and "backward" rays in the linear maser. In all cases, we verify that the results are unaltered when changes are made in the number of rays, the number of locations at which $\rho(\tilde{x}, t)$ is calculated, and the step size used in solving equation (5). For a specified amount of computer time, we find that optimal accuracy is achieved when the separation between the locations at which the $\rho(\tilde{\boldsymbol{x}}, t)$ are computed is much greater (at least a factor of 10) than the step size used in solving equation (5) for the intensities. At intermediate locations, the $\rho(\tilde{\boldsymbol{x}}, t)$ are obtained by interpolation. In the computations for the rectangular parallelepiped, the $\rho(\tilde{x}, t)$ are computed at the grid points of an $8 \times 8 \times 16$ lattice. For the sphere, the only unstable modes that are identified are spherically symmetric. The results presented here are thus based on computations in which $\rho(\tilde{x}, t)$ is found only at a number (24) of radial locations. Exploratory computations have also been performed in which radial symmetry is not imposed. Unstable, nonradial modes have not been found. The sphere is inscribed within a $24 \times 24 \times 24$ cubic grid of lattice points at which the populations $\rho(\tilde{x}, t)$ are computed. A sphere having a diameter equal to the length of an edge of the cubic grid is created by setting the populations $\rho(\tilde{\boldsymbol{x}}, t)$ and $\rho_{\mathrm{s}}$ to zero outside of this spherical volume. As a guideline, the approximate number of rays in a group that is considered to perform the angular integral at each location in equation (1) is the number of lattice points on the surface of the rectangular parallelepiped or on the surface of the cube. That is, approximately 640 (for the $8 \times 8 \times 16$ grid) and 3456 (for the $24 \times 24 \times 24$ grid), respectively. Symmetries can often be utilized to reduce this number. As in SW2, the evolution of the system is initiated by instantaneously "turning on" the pumping. That is, equations (1) and (5) are solved beginning with $\rho(\tilde{x}, t=0)=0$ and $\tilde{\boldsymbol{T}}(\tilde{\boldsymbol{x}}, t=0)=1$ everywhere. How the time evolution is begun was found to be unimportant in SW2 for identifying instabilities and this starting condition is computationally most efficient. After a long time and regardless of the starting conditions, it was found that the radiation from the unstable masers oscillates and that from the stable masers becomes constant. Based on this criterion, complete agreement is demonstrated in SW2 between the results obtained from the time evolution and from the independent, linear stability analysis with regard to which masers are stable and which are unstable.

\section{RESULTS}

Computations are performed for spherical masers and for elongated masers as represented by rectangular parallelepipeds with square cross sections. The ratio of the lengths to the widths of these rectangular parallelepipeds is 100 . These are intended to represent the extremes of the likely geometries. The computations are quite time-consuming, and considerable care must be taken to assure convergence. Only limited results are presented $(a)$ to establish that the basic instability does occur for three-dimensional geometries and $(b)$ to indicate that the values for the parameters at which the instability occurs are similar to those at which it occurs in the linear masers.

The occurrence of permanent oscillations for the emergent maser radiation in Figures 1, 2, and 3 establishes that both

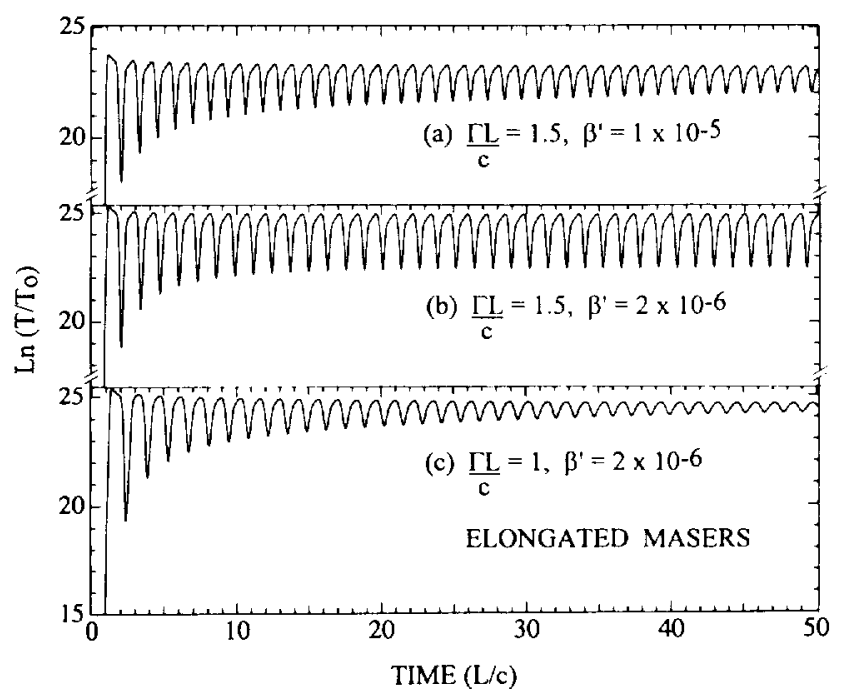

FIG 1-The emergent intensity (as represented by the natural log of the normalized brightness temperature) vs. time for the ray of radiation that is along the axis of an elongated, three-dimensional maser in the form of a rectangular parallelepiped with a square cross section. Time is expressed in units of $L / c$, where $L$ is length of the maser. The maser has a length to width ratio of 100 . Results are shown for three choices of the relevant parameters. Curve $b$ represents an unstable maser; curve $c$, a stable maser and curve $a, a$ barely stable maser. 


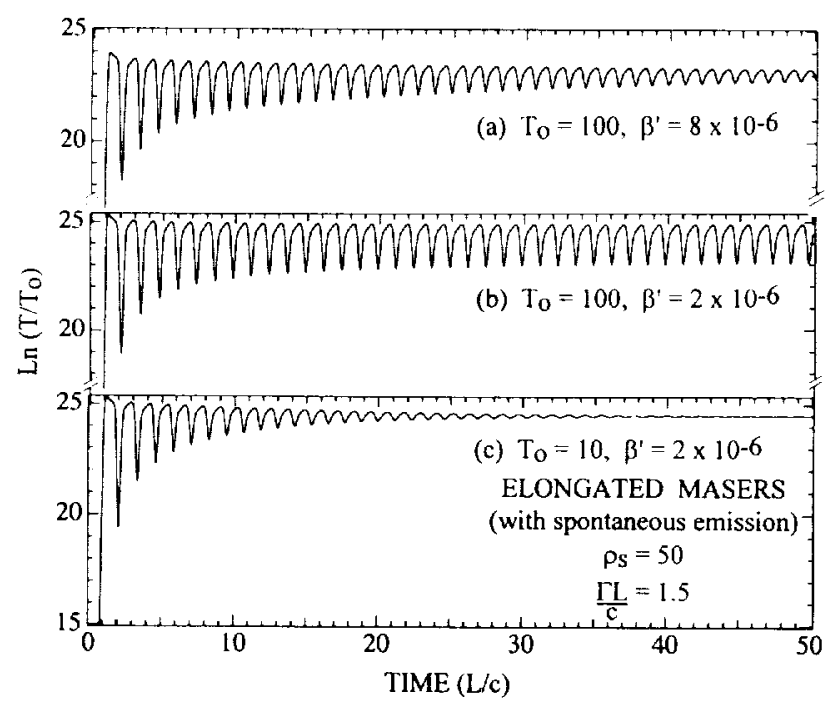

Fig. 2.-The emergent intensity of maser radiation as a function of time, with the same meaning as Fig. 1, except that the influence of spontaneous emission is examined. The importance of spontaneous emission depends upon the parameters $\rho_{s}$ and $T_{0}$

elongated and spherical masers are subject to the instability identified in SW1 and SW2. The intensities are presented in the figures for only a single, representative ray of radiation. For other rays, the variations are similar and have the same phase. As for linear masers, the instability occurs when $\Gamma L / c \simeq 1$ (where $L$ is the length of the elongated masers or the diameter of the spheres) and causes oscillations with a period of approximately $L / c$. Comparison of curves $\mathrm{b}$ and $\mathrm{c}$ in Figure 1 indicates that the instability also disappears at other $\Gamma L / c$. The parameter $\beta^{\prime}$ here is analogous to $\beta$ in SW1 and SW2. Just as for the linear masers, the instability disappears for larger values of this parameter. For the $\mathrm{OH}$ masers at $1665 \mathrm{MHz}$, $\beta^{\prime}=1.4 \times 10^{-5}\left(T_{0} / 100 \mathrm{~K}\right)\left(0.001 \mathrm{~s}^{-1} / \Gamma\right)$. Plausible values for $T_{0}$ and $\Gamma$ (e.g., SW1 and SW2) are such that $\beta^{\prime}$ can be less than $10^{-5}$ as is indicated for instability in Figure 1. Spontaneous emission was previously found to reduce the tendency for instability in linear masers. It has the same effect in threedimensional masers as can be seen by comparing curve $b$ of Figure 2 with curve $b$ in Figure 1. The relative importance of spontaneous emission decreases with decreasing fractional inversion in the pumping - a $1 \%$ difference between the

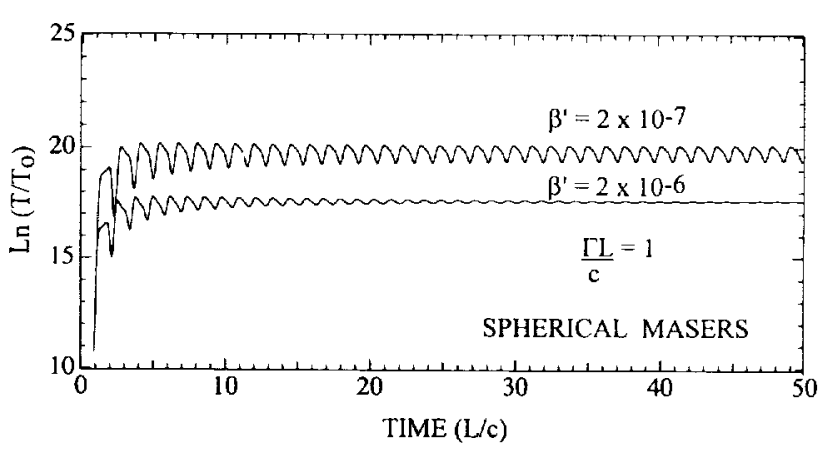

Fic. 3.- Same as Figs. 1 and 2, except that here the maser is in the form of a sphere for which the characteristic length $L$ is the diameter of the sphere. The intensity is shown for a ray that emerges along a diameter.

pumping rates of the upper and lower states corresponds to $\rho_{s}=50$ and is a typical estimate for the $1665 \mathrm{MHz}$ masers as is $T_{0} \simeq 10-100$ (e.g., SW1 and SW2). The diagonal ray between opposite corners of rectangular parallelepipeds is longer, and thus tends to be more intense, than the ray along the axis. For the aspect ratio (100) of the parallelepipeds in Figures 1 and 2, the difference is negligible. This ray is, of course, included in the computations. In exploratory computations for cubic and other rectangular parallelepipeds with lower aspect ratios, the instability persists and the time variations are similar in character to those presented in Figures 1 and 2. Obtaining convergence is more difficult for spherical massers than for elongated masers. The two computations are thus presented in Figure 3 only to establish the principle that spherical masers are also subject to the same instability. The stable solution is presented to demonstrate that our computational methods also lead to stable spherical masers and that this occurs for parameter values at which it would be expected by analogy with linear masers (here, at larger $\beta^{\prime}$ ). Spherical masers tend to be more stable than elongated masers. For the instability of spherical masers, and based upon the information from our computations, $\beta^{\prime}$ must be smaller by at least a factor of 10 than for the elongated masers.

Application of the calculated instabilities to the observations of Clegg \& Cordes (1991) has been discussed in detail in SW2.

This research has been supported in part through NSF grant AST89-19614 and NASA grant NAGW-1104. 